\title{
Formal LPV Control for Transient Stability of Power Systems
}

\author{
Ahmed El-Guindy ${ }^{\star}$, Konstantin Schaab ${ }^{\dagger}$, Bastian Schürmann ${ }^{\star}$ \\ Olaf Stursberg ${ }^{\dagger}$, and Matthias Althoff ${ }^{\star}$ \\ * Department of Informatics, Technical University of Munich (Germany) \\ ${ }^{\dagger}$ Control and Systems Theory, EECS, University of Kassel (Germany)
}

\begin{abstract}
Transient stability analysis of synchronous generators is important for a secure operation of power systems. This paper presents the design and verification of linear-parametervarying (LPV) controllers to robustly establish transient stability of multi-machine power systems with formal guarantees. First, we transform power systems described by differential algebraic equations (DAEs) into modular LPV systems, such that the interaction and correlation between different machines connected to the grid is preserved. Then, we employ reachability analysis to determine the set of admissible parameter values which is required for the LPV controller synthesis. Afterwards, reachability analysis is also used to formally guarantee that the synthesized controller encloses the time-varying parameters within chosen parameter ranges during transients. Both tasks are solved simultaneously in a systematic fashion. The method is demonstrated on a multi-machine benchmark example to showcase the applicability and scalability of the approach.
\end{abstract}

\section{INTRODUCTION}

Transient stability analysis is considered by both theorists and practitioners to be one of the most problematic issues for studies involving the dynamic security assessment of power systems [1]. The problem refers to the ability of the synchronous generator to remain in synchronism with the frequency of the utility grid following a disturbance in the transmission network. This problem can be effectively managed using fast circuit breakers and by introducing necessary damping torque via special controllers, e.g. the power system stabilizer (PSS). However, the standard controllers which establish transient stability in practice are often synthesized based on a linearized model of the synchronous machine [1]-[3]. This limits their effectiveness to eliminate the electrochemical oscillations during transients, around a nearby region from the linearization point. This will become even more challenging in the foreseeable future due to increasingly varying operating conditions in power systems.

An alternative approach to handling nonlinearities of power systems is the use of linear-parameter-varying (LPV) controllers, see e.g. [4]-[6]. The interesting feature about LPV systems is that they can be treated as linear timeinvariant (LTI) plants subject to the uncertainty of some time-varying parameters which account for the operational range of the system. This makes it possible to use powerful linear controller synthesis tools, e.g. robust $\mathcal{H}_{\infty}$ and pole placement [7], [8]. One aspect which is ignored during the synthesis procedure is the verification of the resulting controller; that is, the formal guarantee that the time-varying parameters will always remain within the specified space using the synthesized controller under all eventualities. In other work, the controller is instead examined within a simulation environment that does not provide any guarantees.

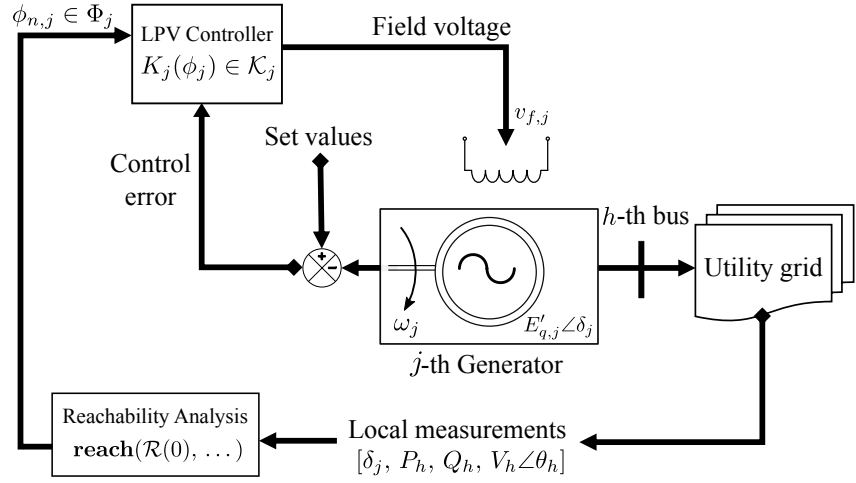

Fig. 1. Simplified diagram of the proposed LPV controller to robustly establish transient stability with formal guarantees for multi-machine power systems. In this framework, the $j$-th generator is controlled via a set of statefeedback controllers $K_{j}\left(\phi_{j}\right) \in \mathcal{K}_{j}$, as presented in Sec. III. The controller gain generates the control signal of the field voltage $v_{f}$, depending on the time-varying parameters $\phi_{n, j} \in \Phi_{j}$ obtained using reachability analysis in Sec. IV. The interaction at the bus $h$ with other machines connected to the grid is preserved by considering local measurements that are affected following any disturbance occurring in the transmission network.

Motivated by the shortcomings of standard controllers and the existing LPV controller synthesis procedure, we present an approach, based on reachability analysis, to combine synthesis and verification of LPV controllers under one framework. Reachability analysis basically determines the set of states that a system can reach over a time horizon starting from a set of initial states [9], and recently, it has emerged as a promising technique for a wide range of applications in power systems, see e.g. [10]-[13]. The proposed framework is particularly beneficial when synthesizing LPV controllers of multi-machine power systems, since finding consistent parameter ranges for each generator simultaneously in the least conservative way can become a difficult task when not following a systematic approach.

\section{Modelling OF LPV POWER Systems}

We consider a standard model of the synchronous generator for the multi-machine power system model [2, p.334]

$$
\begin{aligned}
d \delta_{j} / d t & =\omega_{s}\left(\omega_{j}-\omega_{r}\right), \\
d \omega_{j} / d t & =1 / 2 H_{j}\left(P_{m, j}-P_{e, j}-D_{j}\left(\omega_{j}-\omega_{r}\right)\right), \\
d E_{q, j}^{\prime} / d t & =1 / \tau_{d, j}^{\prime}\left(v_{f, j}-E_{q, j}^{\prime}-i_{d, j}\left(X_{d, j}-X_{d, j}^{\prime}\right)\right) .
\end{aligned}
$$

Here $\delta$ is the rotor angle, $\omega$ is the angular velocity, and $E^{\prime}$ is the machine transient voltage. The system inputs are the field voltage $v_{f}$ and the power $P_{m}$. The subscript $j$ corresponds to the $j$-th generator, $m$ and $e$ are the mechanical and electrical components, respectively, and $d$ and $q$ denote the d- and q-axis, associated with Park's transformation. 
The power $P_{e}$ and the stator current $i$ are obtained by solving the following set of algebraic equations:

$$
\begin{aligned}
& 0=v_{q, j}-E_{q, j}^{\prime}+X_{d, j}^{\prime} i_{d, j}, \\
& 0=v_{d, j}-X_{q, j} i_{q, j}, \\
& 0=v_{d, j}-V_{h} \sin \left(\delta_{j}-\theta_{h}\right), \\
& 0=v_{q, j}-V_{h} \cos \left(\delta_{j}-\theta_{h}\right), \\
& 0=P_{e, j}-v_{d, j} i_{d, j}-v_{q, j} i_{q, j}, \\
& 0=Q_{e, j}-v_{q, j} i_{d, j}+v_{d, j} i_{q, j},
\end{aligned}
$$

where $v$ is the machine voltage and $Q$ is the generator reactive power. The voltage $V$ and its phase angle $\theta$ at the bus $h \in \mathcal{B}$ are associated with the grid algebraic equations. They are calculated via the formulation of the power flow equations [2, p.68]

$$
\begin{aligned}
& 0=P_{h}-V_{h} \sum_{k \in \mathcal{B}} V_{k}\left|Y_{h k}\right| \cos \left(\Theta_{h k}-\theta_{k}-\theta_{h}\right), \\
& 0=Q_{h}+V_{h} \sum_{k \in \mathcal{B}} V_{k}\left|Y_{h k}\right| \sin \left(\Theta_{h k}-\theta_{k}-\theta_{h}\right),
\end{aligned}
$$

with the line admittance being expressed by the absolute value $Y$ and the phase angle $\Theta$. The parameters $D, H, \omega_{r}$, $\omega_{s}, X, X^{\prime}$, and $\tau^{\prime}$ are the damping and the inertia constants, the reference and base frequencies, the synchronous and transient reactance, and the transient time constant, respectively.

The LPV system representation of the synchronous generator is derived in [14] by inserting a suitable choice of algebraic variables into the differential equations, and assigning the remaining nonlinearities to time-varying parameters:

$\dot{\tilde{x}}_{j}=\left[\begin{array}{ccc}0 & \omega_{s} & 0 \\ \frac{\phi_{1, j}}{2 H_{j}} & -D_{j} & -\frac{\phi_{2, j}}{2 H_{j}} \\ -\frac{\left(X_{d, j}-X_{d, j}^{\prime}\right) \phi_{3, j}}{\tau_{d, j}^{\prime}} & 0 & -\frac{1}{\tau_{d, j}^{\prime}}\end{array}\right] \tilde{x}_{j}+\left[\begin{array}{c}0 \\ 0 \\ \frac{1}{\tau_{d, j}^{\prime}}\end{array}\right] v_{f, j}$,

with : $\left.\begin{array}{r}\phi_{1, j}=P_{m, j}-\left(X_{q, j}-X_{d, j}^{\prime}\right) i_{d, j} i_{q, j} / \delta_{j}, \\ \phi_{2, j}=i_{q, j}, \phi_{3, j}=i_{d, j} / \delta_{j}\end{array}\right\} \phi=F\left(\delta, i_{d}, i_{q}\right)$

where $\phi_{n, j} \in \Phi_{j}, n \in\left\{1, \ldots, n_{\phi}\right\}$ are the time-varying parameters, and the state variables are $\tilde{x}=\left[\delta, \omega, E_{q}^{\prime}\right]^{T}$. Note that the power $P_{m}$ is constant, which can be easily justified for studies involving transient stability [1, ch.13].

Remark 1. Here it should be stressed that (4) is an exact reformulation and is not a linearization of (1)-(2). This allows one to set up modular models of the generators to synthesize and verify each machine separately.

\section{LPV CONTROLLER SYNTHESIS}

This section describes the synthesis procedure for each $j$-th generator to obtain a set of state-feedback controllers, as illustrated in Fig. 1. First, we express the LPV system (4) in state-space form as

$$
\begin{aligned}
\dot{\tilde{x}}_{j}(t) & =\tilde{A}_{j}\left(\phi_{j}(t)\right) \tilde{x}(t)+B_{j} u_{v, j}(t)+B_{\infty, j} u_{w, j}(t), \\
z_{j}(t) & =C_{\infty, j} \tilde{x}_{j}(t),
\end{aligned}
$$

with $u_{v, j} \in \mathbb{R}^{n_{v}}$, and $u_{w, j} \in \mathbb{R}^{n_{w}}$ as the vector of control outputs and exogenous inputs, respectively, and $z_{j} \in \mathbb{R}^{n_{z}}$ as the output vector to specify the control performance of a transfer function $G_{z_{j} u_{w, j}}(s)$. The system matrix $\tilde{A}_{j}\left(\phi_{j}(t)\right)$ depends affinely on the time-varying parameters, such that

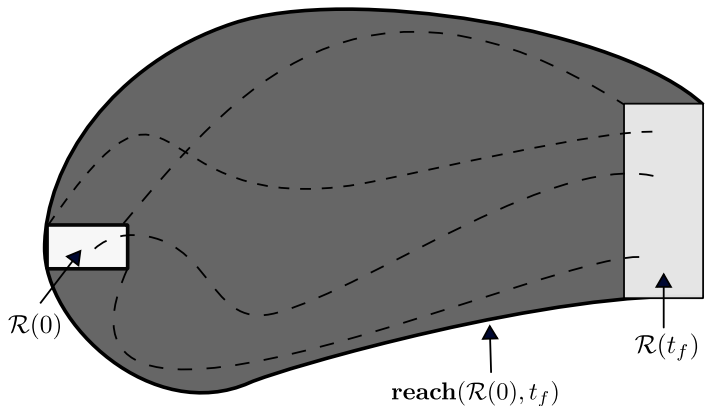

Fig. 2. Simplified illustration of a reachable set. The dark-gray area is the resulting set reach $\left(\mathcal{R}(0), t_{f}\right)$ that encloses all possible system trajectories for $t \in\left[0, t_{f}\right]$, starting from a set of initial states $\mathcal{R}(0)$, over a time horizon $t_{f}$. Random trajectories are shown by dotted-lines. The light-gray area represents the reachable set at $t=t_{f}$ denoted by $\mathcal{R}\left(t_{f}\right)$.

$\tilde{A}_{j}\left(\phi_{j}\right) \in \tilde{\mathcal{A}}_{j}:=\left\{\sum_{i=1}^{l_{\phi}} \lambda_{i, j}\left(\phi_{j}\right) \tilde{A}_{j}^{(i)}: \lambda_{i} \geq 0, \sum_{i=1}^{l_{\phi}} \lambda_{i}=1\right\}$,

where $\tilde{\mathcal{A}}_{j}$ is a matrix-polytope defined as the convex hull of a finite number of matrices with the same dimensions denoted by $\tilde{A}_{j}^{(i)} \in \mathbb{R}^{n_{\tilde{x}} \times n_{\tilde{x}}}, i \in\left\{1, \ldots, l_{\phi}\right\}$ and $\lambda_{i}\left(\phi_{j}\right)$ are the coefficients of the convex combination that expresses the variable matrix $\tilde{A}_{j}\left(\phi_{j}\right)$ in terms of the vertices $\tilde{A}_{j}^{(i)}$.

Next, the set of state-space controllers is obtained by solving a semi-definite optimization problem subject to a set of LMIs specified for $\mathcal{H}_{\infty}$ and pole placement [7], [8]:

$$
\begin{gathered}
\operatorname{minimize}_{M_{j}, \tilde{K}_{j}^{(i)}} \gamma_{j}, \\
2 \alpha_{j} M_{j}+\tilde{A}_{c l, j}^{(i)} M_{j}+M_{j} \tilde{A}_{c l, j}^{(i) T}<0, \\
{\left[\begin{array}{cc}
\sin \left(\beta_{j}\right)\left(T_{1, j}\right) & \cos \left(\beta_{j}\right)\left(T_{2, j}\right) \\
-\cos \left(\beta_{j}\right)\left(T_{2, j}\right) & \sin \left(\beta_{j}\right)\left(T_{1, j}\right)
\end{array}\right]<0,} \\
{\left[\begin{array}{ccc}
\tilde{A}_{c l, j}^{(i)} M_{j}+M_{j} \tilde{A}_{c l, j}^{(i) T} & B_{\infty, j}^{T} & M_{j} C_{\infty, j}^{T} \\
B_{\infty, j}^{T} & -\gamma_{j} I & 0 \\
C_{\infty, j}^{T} & 0 & -\gamma_{j} I
\end{array}\right]<0,} \\
\text { with : } \tilde{A}_{c l, j}^{(i)}:=\tilde{A}_{j}^{(i)}+B_{j} \tilde{K}_{j}^{(i)},
\end{gathered}
$$

where $\tilde{K}_{j}^{(i)}$ are the controller vertices and $M_{j}$ is the symmetric matrix of the Lyapunov function $V_{j}\left(\tilde{x}_{j}\right)=\tilde{x}_{j}^{T} M_{j}^{-1} \tilde{x}_{j}$. The expressions of $T_{1, j}$ and $T_{2, j}$ are provided in [14].

The constraint conditions (9), (10), and (11) physically account for limiting the energy consumption of the actuating variable while introducing sufficient damping torque via the exciter field voltage $v_{f, j}$ in order to stabilize the $j$-th synchronous machine during transients. This is achieved via the parameters $\gamma_{j}, \alpha_{j}$, and $\beta_{j}$, whose physical meaning is:

- The parameters $\alpha_{j}$ and $\beta_{j}$ are associated with the pole placement design. They define a region realized with $\operatorname{Re}(s)>-\alpha_{j}$ and a conic sector with the angle $\beta_{j}$ which specifies the pole locations of the closed-loop within the complex plane $s$, thus directly influencing the dynamic performance.

- The parameter $\gamma_{j}$ is the bound that accounts for the closed-loop $\mathcal{H}_{\infty}$ performance such that $\left\|G_{z_{j} u_{w, j}}\right\|_{\infty}<$ $\gamma_{j}$, i.e. this condition addresses the controller robustness. 
Finally, the controller $K_{j}\left(\phi_{j}\right)$ can be expressed analytically during control using the convex combination based on the controller vertices $\tilde{K}^{(i)}$, such that

$$
K_{j}\left(\phi_{j}\right)=\sum_{i=1}^{l_{\phi}} \lambda_{i, j}\left(\phi_{j}\right) \tilde{K}_{j}^{(i)},
$$

where the coefficients $\lambda_{i, j}\left(\phi_{j}\right)$ are the same as those appearing in (7), due to the affine dependency of the LPV controller on the time-varying parameters.

In Sec. IV-C, we will illustrate that time-varying parameters are enclosed by an axis-aligned box $\Phi:=[\underline{\phi}, \bar{\phi}]$ with the extreme points $\hat{\phi}_{1}, \ldots, \hat{\phi}_{2^{n} \phi}$, where $\phi$ and $\bar{\phi}$ are the lower and upper bound of time-varying parameters, correspondingly. This makes it possible to express the dependency of the coefficients $\lambda_{i}$ on $\phi$ by the closed-form expression

$$
\lambda_{i}(\phi)=\prod_{m=1}^{n_{\phi}} \bar{\nu}_{i, m}(\phi),
$$

with $i$ and $m$ denoting the $i$-th entry and the $m$-th dimension, respectively. The formula of the variable $\bar{\nu}$ and the proof of (13) are detailed in our previous work [15].

\section{ReACHABILITY ANALYSIS}

This section illustrates the procedure to obtain the set of parameter ranges $\Phi$ necessary for the LPV controller synthesis based on computations of over-approximative reachable sets. Reachability analysis of dynamical systems determines the set of states that a system can reach over a time horizon starting from a set of initial states, as illustrated in Fig. 2.

\section{A. Abstraction to Linear Differential Inclusions}

First, we rewrite the multi-machine power system model in (1)-(3) using the compact form formalized as set of timeinvariant, semi-explicit, nonlinear, index-1 DAEs

$$
\begin{aligned}
\dot{x}(t) & =f(x(t), y(t), u(t)), \\
0 & =g(x(t), y(t), u(t)),
\end{aligned}
$$

where the vectors $x \in \mathbb{R}^{n_{x}}, y \in \mathbb{R}^{n_{y}}$, and $u \in \mathbb{R}^{n_{u}}$ include the dynamic states, the algebraic constraints, and the input variables, respectively. Our reachability algorithm is based on abstracting (14) into linear differential inclusions for each consecutive time-interval $\tau_{k}:=\left[t_{k}, t_{k+1}\right]$, with $t_{k}:=k r$, where $r \in \mathbb{R}^{+}$refers to the step size, and $k \in\{1, \ldots, h\}$ is the time step. The abstraction is expressed by

$$
\forall t \in\left[t_{k}, t_{k+1}\right]: \dot{x}(t) \in A x(t) \oplus \mathcal{U} .
$$

Here $A \in \mathbb{R}^{n_{x} \times n_{x}}$ is the abstraction system matrix, and $\mathcal{U}$ is the set of uncertain inputs. The operator $\oplus$ returns the Minkowski sum of two sets.

Remark 2. Note that (14) is continuously linearized for each time interval $\tau_{k}$. Additionally, the inclusion (15) encloses all possible trajectories of the nonlinear DAE system.

\section{B. Computation of Over-Approximative Reachable Sets}

After defining $r:=t_{k+1}-t_{k}$ and $u_{c}$ as the center of $\mathcal{U}$, we can express the reachable set $\mathcal{X}\left(t_{k+1}\right)$ of the dynamics $\dot{x}=$ $A_{k} x+u_{c}$, enclosed by the differential inclusion (15), based on the well-known solution of linear state-space equations

$$
\mathcal{X}\left(t_{k+1}\right)=e^{A_{k} r} \mathcal{R}\left(t_{k}\right) \oplus \mathcal{R}_{p}(r),
$$

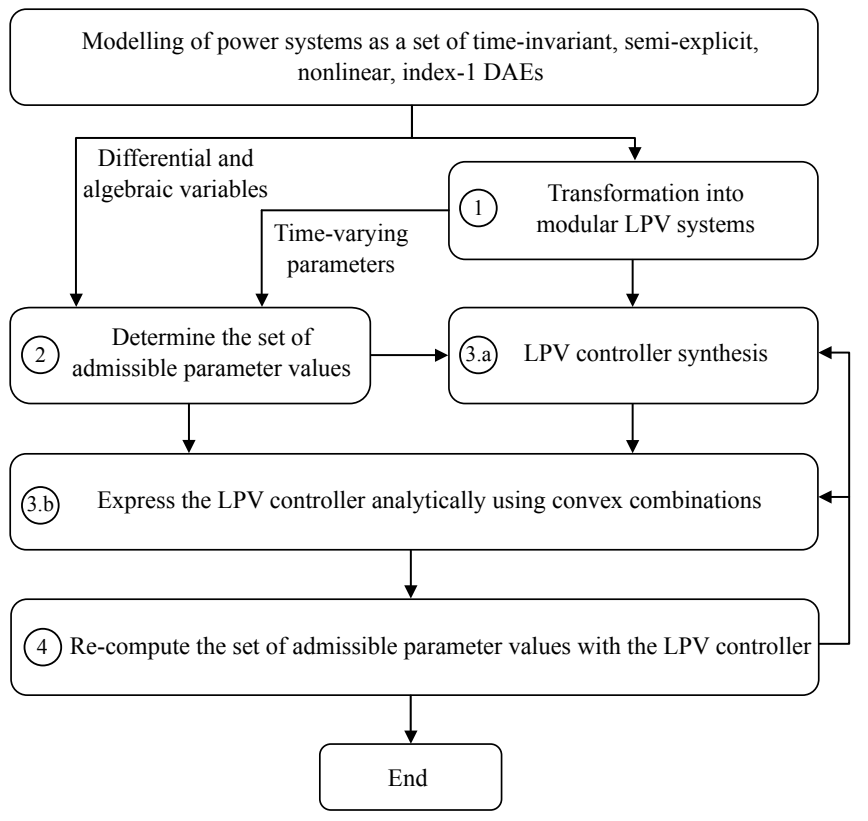

Fig. 3. Illustration of the proposed approach to combine synthesis and verification of LPV control under one framework, to robustly establish transient stability of multi-machine power systems with formal guarantees.

where $e^{A_{k} r}$ is the matrix exponential and $\mathcal{R}_{p}(r)$ is the set which over-approximates the particular solution of the linear state-space equation. The reachable sets at the next point in time $t_{k+1}$, and for the time interval $\tau_{k}$, are evaluated by

$$
\begin{aligned}
\mathcal{R}\left(t_{k+1}\right) & =\mathcal{X}\left(t_{k+1}\right) \oplus \mathcal{R}_{u}(r), \\
\mathcal{R}\left(\tau_{k}\right) & =\mathbf{C H}\left(\mathcal{R}\left(t_{k}\right), \mathcal{X}\left(t_{k+1}\right)\right) \oplus \mathcal{R}_{e} \oplus \mathcal{R}_{u}(r),
\end{aligned}
$$

where the operator $\mathbf{C H}(\cdot)$ returns the convex hull of two sets, and the set $\mathcal{R}_{e}$ considers enlargement of the convex hull enclosure, in order to account for the assumption that trajectories over $\tau_{k}$ are straight lines. Finally, the reachable set $\mathcal{R}_{u}(r)$ accounts for the uncertainty of the input set $\mathcal{U}$. The evaluation of the sets $\mathcal{R}_{p}(r), \mathcal{R}_{e}$, and $\mathcal{R}_{u}(r)$ are derived in our previous work [12].

\section{Reachable Set of Time-Varying Parameters}

To estimate the admissible set of time-varying parameters, we over-approximate computed sets (17), (18) using

$$
\begin{aligned}
\eta_{k}^{d} & :=\mathbf{I H}\left(\mathcal{R}^{d}\left(\tau_{k}\right)\right)=\left[\underline{\eta}_{k}^{d}, \bar{\eta}_{k}^{d}\right], \\
\eta_{k}^{a} & :=\mathbf{I H}\left(\mathcal{R}^{a}\left(\tau_{k}\right)\right)=\left[\underline{\eta}_{k}^{a}, \bar{\eta}_{k}^{a}\right],
\end{aligned}
$$

where $\eta_{k}$ is the interval hull which approximates computed reachable sets. The superscripts $d$ and $a$ corresponds to differential and algebraic variables. The operator $\mathbf{I H}(\cdot)$ returns the interval that encloses a set as tightly as possible.

The interval hulls $\boldsymbol{X}$ and $\boldsymbol{Y}$ enclosing the evolution of differential and algebraic variables for (14) over a timehorizon $t_{f}$ with $h:=t_{f} / r$ time-steps, are

$$
\begin{aligned}
\boldsymbol{X} & :=\operatorname{enclose}\left(\eta_{k}^{d}, \ldots, \eta_{h}^{d}\right)=[\underline{x}, \bar{x}], \\
\boldsymbol{Y} & :=\operatorname{enclose}\left(\eta_{k}^{a}, \ldots, \eta_{h}^{a}\right)=[\underline{y}, \bar{y}],
\end{aligned}
$$




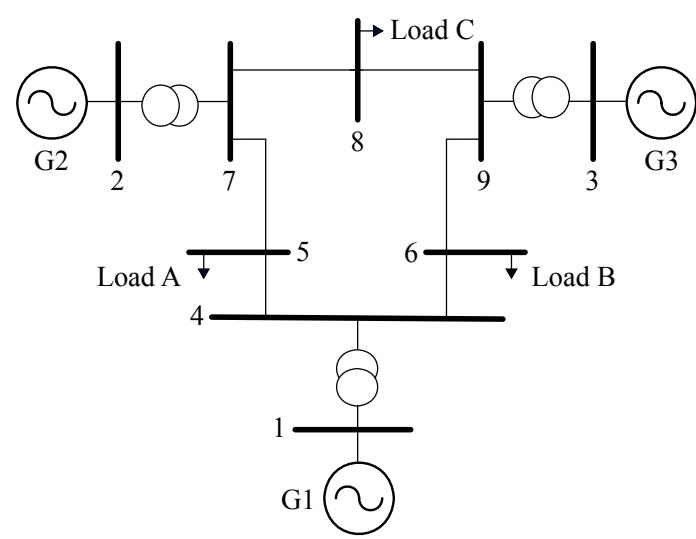

Fig. 4. The WSCC 3-machine 9-bus benchmark [3, Ch. 2].

$$
\begin{aligned}
\text { using } \quad \underline{x} & :=\min _{k \in\{1 \ldots h\}} \underline{\eta}_{k}^{d}, \text { and } \bar{x}:=\max _{k \in\{1 \ldots h\}} \bar{\eta}_{k}^{d}, \\
\underline{y} & :=\min _{k \in\{1 \ldots h\}} \underline{\eta}_{k}^{a}, \text { and } \bar{y}:=\max _{k \in\{1 \ldots h\}} \bar{\eta}_{k}^{a},
\end{aligned}
$$

therefore, the set of admissible parameter values of (6) over a time-horizon $t_{f}$ can be expressed by

$$
\Phi_{j}=\left\{\phi_{j}=\boldsymbol{F}_{j}(x, y): x \in \boldsymbol{X}, y \in \boldsymbol{Y}\right\} .
$$

Here, $\boldsymbol{F}(x, y)$ is the nonlinear function that describes timevarying parameters as in (5). The function is evaluated using interval arithmetic since differential and algebraic variables are presented by interval vectors in (20).

\section{OVERALl APPROACH}

Combining all previous steps, we can now summarize the overall original approach to synthesizing LPV controllers with formal guarantees. The procedure shown in Fig. 3 is outlined as follows

(1) Transform each grid node of the multi-machine power system DAE model (14), which corresponds to a generator bus to the LPV representation (4).

(2) Perform transient stability analysis on the DAE system (14) using reachability algorithms to guess the set of parameter values $\Phi_{j}$ for each $j$-th generator.

3.a Synthesize a set of controllers $K_{j}\left(\phi_{j}\right) \in \mathcal{K}_{j}$ that account for admissible parameter trajectories obtained in (2) by formulating a set of LMIs specified for pole placement and $\mathcal{H}_{\infty}$ design.

(3.b) Express the LPV controller analytically based on the synthesized set of state-space controllers.

(4) Verify the design specifications of the controller using reachability analysis, to guarantee that the time-varying parameters are always enclosed within the specified admissible space.

\section{CASE STUdy}

We consider the multi-machine WSCC 9-bus benchmark example [3, Ch. 2], illustrated in Fig. 4. We assume the PQ-loads and transformers are modeled as constant impedances. The LPV controller is synthesized around an LMI-region consisting of the half-plane $\operatorname{Re}(s)>-7$ and the conic sector with an angle $\beta=45^{\circ}$. This enforces sufficient damping of approximately $70 \%$, thus eliminating the
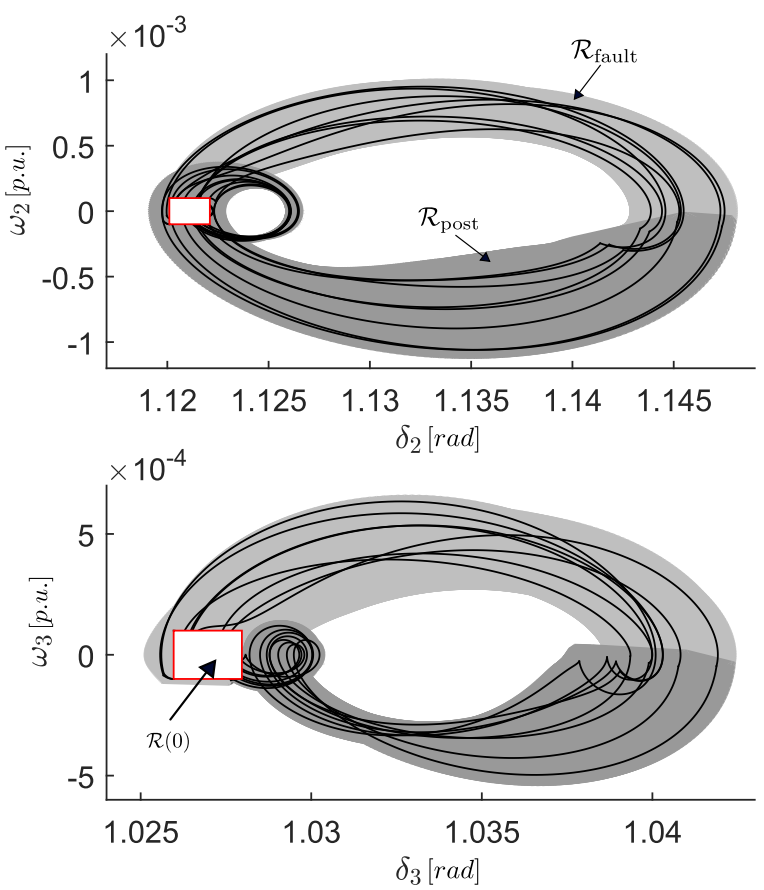

Fig. 5. Projection of the reachable set of differential variables for generators $G_{2}$ and $G_{3}$. The light-gray and dark-gray areas belong to the reachable set during fault and post-fault, respectively. The white box corresponds to the set of initial state variables $\mathcal{R}(0)$. The considered fault scenario is the loss of the transmission line connecting the buses 5 and 7 . The line is reconnected after the clearance of the fault, and the reachable set is computed until all states are enclosed by $\mathcal{R}(0)$, to formally verify that the LPV controller introduces sufficient damping torque to converge state variables back the original equilibrium point. The solid lines present random simulation results starting from the initial reachable set $\mathcal{R}(0)$.

electromechanical oscillations in a reasonable time. Table I provides the admissible space of $\Phi_{j}$ for each $j$-th generator, which is required for the controller synthesis procedure.

The considered fault scenario is the loss of the transmission line between bus 5 and 7 . Immediately after losing the transmission line, entries of the admittance matrix change thus leading to a discontinuous jump in the algebraic variable to satisfy the power flow equations (3). This disturbance generates a new control action from the unified control structure, see Fig. 1, as the local measurements $\delta, P, Q, V \angle \theta$ at each generator bus were affected by the perturbation in the transmission network. Fig. 5 shows projection of the reachable set using the synthesized LPV controller for the considered fault scenario.

We include uncertainty in the initial set of differential variables, since initial states are not exactly known due to

TABLE I

ADMISSIBLE SPACE OF THE TIME-VARYING PARAMETERS FOR EACH GENERATOR OF THE WSCC 9-BUS BENCHMARK EXAMPLE.

\begin{tabular}{cccc}
\hline$\Phi$ & $G_{1}$ & $G_{2}$ & $G_{3}$ \\
\hline$\Phi_{1}$ & {$[0.21,0.25]$} & {$[0.04,0.06]$} & {$[0.07,0.11]$} \\
$\Phi_{2}$ & {$[0.25,0.33]$} & {$[0.84,1.12]$} & {$[0.51,0.62]$} \\
$\Phi_{3}$ & {$[0.34,18.9]$} & {$[1.19,1.68]$} & {$[0.46,0.59]$}
\end{tabular}



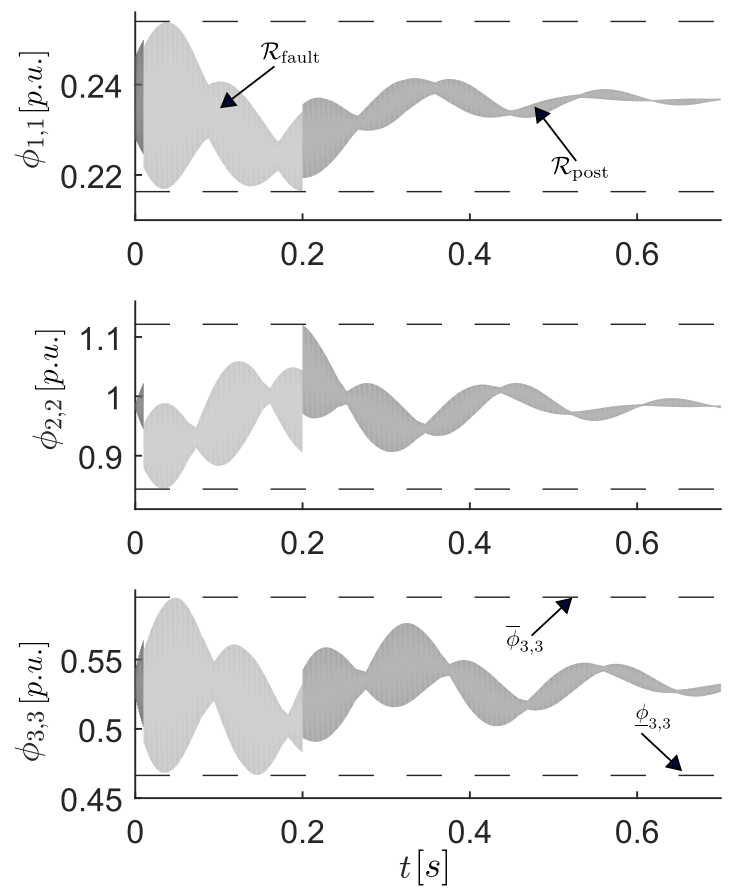

Fig. 6. Time-domain bounds of chosen time-varying parameters. The light-gray and dark-gray areas belong to the reachable set during fault and post-fault, respectively. The jump in $\phi_{2,2}$ at $t=0.01$, and $t=0.2$ is associated with the fault scenario which leads to the discontinuous change in the reachable set. The dashed-lines are the upper and lower bounds of the time-varying parameters, see Table I. Since the time-domain bounds do not intersect with the dashed-lines, it can be formally verified that the controller robustly establish transient stability according to its design specifications.

increasingly varying operating conditions in current power systems. Note that the post-fault phase is analyzed until all dynamic state variables converge back to the initial set denoted by $\mathcal{R}(0)$. To examine accuracy and tightness of the over-approximative reachable set, we validate the results against simulated trajectories of the DAE system starting from the set of initial states $\mathcal{R}(0)$. It can be seen that the reachable sets tightly enclose all trajectories, thus allowing one to estimate the set of admissible parameter values in the least conservative way. The parameters are obtained via the computation of reachable sets of differential and algebraic variables according to the method explained in Sec. IV-C. Reachability analysis is also used to verify that these bounds are not violated using the synthesized controller for the considered fault scenario, as shown in Fig. 6.

Comparing our results with an existing method is not possible due to the originality of the proposed method. A comparison, however, is indeed possible when considering each aspect separately, i.e. controller synthesis or verification, as reported in our previous work [12]-[14]. A comparison of the LPV control performance against the standard PSS controller is found in [14], where it is shown that the LPV controller outperforms the PSS in many aspects, improving the control performance significantly and yielding much tighter reference value tracking during transients. Furthermore in [12], [13], we highlight the many advantages of reachability analysis over numerical simulations, and present the verification of state-space controllers in a realistic configuration of a power plant.

\section{CONCLUSION}

We propose a unified approach based on reachability analysis to combine synthesis and verification LPV controllers in one framework, to robustly establish transient stability of multi-machine power systems with formal guarantees. The proposed approach reformulates the set of nonlinear DAEs governing dynamics of power systems into modular LPV systems, thus allowing one to systematically synthesize and verify decentralized controllers, and more importantly preserve the correlation between different machines connected to the grid. In future work, the concept applicability will be studied and validated against a realistic setup employing a standard controller, e.g. the PSS, belonging to the $450 \mathrm{MW}$ power plant München Süd GuD, owned by Munich City Utilities, similar to our recent contribution in this area [13].

\section{ACKNOWLEDGMENT}

The authors gratefully acknowledge financial support by the German Research Foundation (DFG) project ROCS-Grid, and the European Commission project UnCoVerCPS 643921. We would also like to express our gratitude to the reviewers for their constructive feedback.

\section{REFERENCES}

[1] P. Kundur, N. J. Balu, and M. G. Lauby, Power system stability and control. McGraw-hill New York, 1994, vol. 7.

[2] F. Milano, Power system modelling and scripting. Springer Science \& Business Media, 2010.

[3] P. M. Anderson and A. A. Fouad, Power System Control and Stability. Wiley-IEEE Press, 2002.

[4] R. He, K.-Z. Liu, and S. Mei, "LPV modelling and gain-scheduled control approach for the transient stabilization of power systems," IEEJ Transactions on Electrical and Electronic Engineering, vol. 5, no. 1, pp. 87-95, 2010.

[5] Q. Liu, V. Vittal, and N. Elia, "LPV supplementary damping controller design for a thyristor controlled series capacitor (TCSC) device," IEEE Transactions on Power Systems, vol. 21, no. 3, pp. 1242-1249, 2006.

[6] W. Qiu, V. Vittal, and M. Khammash, "Decentralized power system stabilizer design using linear parameter varying approach," IEEE Transactions on Power Systems, vol. 19, no. 4, pp. 1951-1960, 2004.

[7] P. Apkarian, P. Gahinet, and G. Becker, "Self-scheduled $\mathcal{H}_{\infty}$ control of linear parameter-varying systems: A design example," Automatica, vol. 31, no. 9, pp. 1251-1261, 1995.

[8] M. Chilali and P. Gahinet, " $\mathcal{H}_{\infty}$ design with pole placement constraints: an LMI approach," IEEE Transactions on Automatic Control, vol. 41, no. 3, pp. 358-367, 1996.

[9] C. Le Guernic, "Reachability analysis of hybrid systems with linear continuous dynamics," Ph.D. dissertation, Université Joseph-FourierGrenoble I, 2009.

[10] Y. C. Chen and A. D. Domínguez-García, "A method to study the effect of renewable resource variability on power system dynamics," IEEE Transactions on Power Systems, vol. 27, no. 4, pp. 1978-1989, 2012.

[11] A. El-Guindy, Y. C. Chen, and M. Althoff, "Compositional transient stability analysis of power systems via the computation of reachable sets," in Proc. of the IEEE American Control Conference, 2017.

[12] M. Althoff, "Formal and compositional analysis of power systems using reachable sets," IEEE Transactions on Power Systems, vol. 29, no. 5, pp. 2270-2280, 2014.

[13] A. El-Guindy, D. Han, and M. Althoff, "Formal analysis of drumboiler units to maximize the load-following capabilities of power plants," IEEE Transactions on Power Systems, vol. 31, no. 6, pp. 46914702, 2016.

[14] K. Schaab and O. Stursberg, "Robust decentralized LPV control for transient stability of power systems," in Proc. of the 9th IFAC Symp. on Control of Power and Energy Systems, 2015, pp. 566-571.

[15] B. Schürmann, A. El-Guindy, and M. Althoff, "Closed-form expressions of convex combinations for controller design," in Proc. of the 2016 IEEE American Control Conference, 2016, pp. 2795 - 2801. 